\title{
Establishment and verification of prediction models for evaluating the physical and chemical properties of soilless substrates
}

\author{
Binbin Gong ${ }^{1 \dagger}$, Ning Wang ${ }^{1 \dagger}$, Tiejun Zhang ${ }^{2}$, Shao $\mathrm{Li}^{3}$, Xiaolei Wu ${ }^{1}$, Jing $\operatorname{Tian}^{3}$, \\ Jingrui $\mathrm{Li}^{1}$, Guiyun Lyu ${ }^{1}$, Hongbo Gao ${ }^{1 *}$ \\ (1. College of Horticulture, Hebei Agricultural University, Baoding 071001, Hebei, China; \\ 2. Modern Education Technology Center, Hebei Agricultural University, Baoding 071001, Hebei, China; \\ 3. Academy of Agricultural Planning and Engineering, MARA, Beijing 100125, China)
}

\begin{abstract}
In soilless culture, a suitable mixed substrate that provides a balanced and stable rhizosphere environment is vital for promoting plant growth. The present study was undertaken to establish seven prediction models of physical and chemical properties, including bulk density (DB), total porosity (TP), water-holding porosity (WHP), air porosity (AP), WHP/AP, electrical conductivity (EC) and cation exchange capacity (CEC) of mixed substrate based on regression equations of measured values from 76 substrate combinations. These seven models were verified using the measured values of 12 mixed substrates, and the average relative prediction errors (REs) were all less than $10 \%$. A comprehensive property prediction model was established by weighted summation of the seven models of physical and chemical properties. According to the set values of $\mathrm{DB}, \mathrm{TP}, \mathrm{WHP}, \mathrm{AP}, \mathrm{WHP} / \mathrm{AP}, \mathrm{EC}$ and CEC, the comprehensive property model predicted the six mixture proportions of mixed-substrate, as verified using the measured values. This study is the first to establish prediction models for the physical and chemical properties of mixed substrates. The comprehensive property model could be used to evaluate the physical and chemical properties of commercial mixed substrates, and to provide the optimal mixture substrate formulations according to the setting property value of production requirement.
\end{abstract}

Keywords: prediction model, mixed substrate, physical and chemical properties, multiple regressions, genetic algorithm DOI: $10.25165 /$ j.ijabe.20211402.5815

Citation: Gong B B, Wang N, Zhang T J, Li S, Wu X L, Tian J, et al. Establishment and verification of prediction models for evaluating the physical and chemical properties of soilless substrates. Int J Agric \& Biol Eng, 2021; 14(2): 9-18.

\section{Introduction}

Soilless plant culture is a cultivation method that does not use soil to support the plant growth, among which mainly involves containerization of plant roots within porous rooting medium known as 'substrate' or 'growing media' ${ }^{[1]}$. Compared with soil-based cultivation, the suitable substrate can provide a balanced and stable rhizosphere environment containing water, gas and fertilizer as well as in the absence of weeds, insects, and pathogens, which is the most important to promote plant growth and improve yield or quality ${ }^{[2]}$. As a result, commercial soilless substrate productions have become increasingly important for plug-seedlings as well as the production of agronomic and horticultural crop species over the last 50 years ${ }^{[3]}$.

Nowadays, with continuous expansion of the substrate

\section{Received date: 2020-04-01 Accepted date: 2020-07-13}

Biographies: Binbin Gong, MS, research interests: greenhouse environment engineering and soilless culture, Email: yygbb@hebau.edu.cn; Ning Wang, MS, research interests: soilless culture, Email: 524590452@qq.com; Tiejun Zhang, Researcher, research interests: automatic control of protected horticulture, Email: zhangtj@hebau.edu.cn; Shao Li, PhD, research interests: soilless facilities, Email: lishao@aape.org.cn; Xiaolei Wu, PhD, research interests: protected vegetable and soilless culture, Email: yywxl@hebau.edu.cn; Jing Tian, PhD, research interests: soilless facilities, Email: tianjing@ aape.org.cn; Jingrui Li, $\mathrm{PhD}$, research interests: protected vegetable and soilless culture, Email: yyljr@hebau.edu.cn; Guiyun Lyu, PhD, research interests: protected vegetable and soilless culture, Email: yylgy@hebau.edu.cn.

$\dagger$ The authors contributed equally to this work.

*Corresponding author: Hongbo Gao, $\mathrm{PhD}$, Professor, research interests: protected vegetable and soilless culture. College of Horticulture, Hebei Agricultural University, Lekai South Street 2596, Baoding 071001, Hebei, China. Tel: +86-312-7528314, Email: hongbogao@hebau.edu.cn. cultivation area, an increasing number of substrate types are being used in crop production, including organic materials such as peat moss, coconut coir, tree bark, biochar and mushroom residue and inorganic materials such as vermiculite, perlite and sand ${ }^{[4-7]}$. According to a recent investigation on substrate utilization in China, the demand for solid substrate annually is approximately 50 million $\mathrm{m}^{3}$, including 15 million $\mathrm{m}^{3}$ for vegetables, 10 million $\mathrm{m}^{3}$ for landscaping, 8 million $\mathrm{m}^{3}$ for rice seedlings, 8 million $\mathrm{m}^{3}$ for soilless cultivation and 9 million $\mathrm{m}^{3}$ for soil restoration ${ }^{[8]}$. Generally, the physical and chemical properties of any substrate employed on its own are limited, leading to difficulties in meeting the needs of crop growth ${ }^{[9,10]}$. Therefore, substrates mixed together at various proportions are widely used in agronomic and horticultural production. During the substrate mixed process, the formulation of different substrate proportion should be adjusted according to the characteristics of used single substrate and different crops to meet the needs of plant growth for the root environment ${ }^{[10]}$. However, there is currently no standardized method to evaluate whether the properties of the mixed substrate can meet the needs of plants, and the design of the substrate mixed formulation is also mainly based on limited data from proportional experiments or farmer's experience ${ }^{[2,11]}$.

Many studies have shown that new substrates offer more option to replace the conventional substrate in physical and chemical properties mainly manifested in suitable water-holding and air-holding capacities as well as ion content and ion adsorption capacity. Vaughn et al. ${ }^{[7]}$ demonstrated that the result of mixed substrate with potato digestate: wood biochar improving growth of tomato plants was better than control substrate of peat: vermiculite; Kuisma et al. ${ }^{[12]}$ evaluated that ground reed canary grass may be used to replace peat or coir in soilless culture of strawberry; Lei et 
al. ${ }^{[13]}$ demonstrated that the substrate of hydroponically grown long-mat seedlings could replace the traditional substrate or nutritive soil in rice seedling production. However, the physical and chemical properties such as bulk density, ion content and ion adsorption capacity of substrates, especially organic substrates, exhibit significant differences among regions ${ }^{[14,15]}$, which sometimes leads to the growth and yield attained with mixed substrates vary obviously under different cultivation practices ${ }^{[16,17]}$. At present, most of commercial mixed substrates sometimes have difficulty to meet the needs of crop varieties, which may lead to slowed growth, reduced yields and other serious consequences ${ }^{[7,18]}$. The main reason is that these mixed formulations were selected based on small-scale experiment or experience may not be optimal; and the experimental results closely relied on the environment of the facility ${ }^{[19,20]}$ and management technology ${ }^{[21,22]}$, which made it difficult to replicate results and apply them in production. Therefore, the investigation of a simple method for predicting the physical and chemical properties of mixed substrates and the mixture proportions of different substrates is necessary to improve the production quality of agronomic and horticultural crops.

Multivariate regression analysis can provide scientific and quantitative information on the relationships among variable, which is widely used in fields involving agricultural production, such as greenhouse irrigation management ${ }^{[23-25]}$, plant nutrient element detection and analysis ${ }^{[26-28]}$, and crop yield analysis ${ }^{[29]}$. Similarly, this technique is also the best method for investigating soilless substrates to date, such as analyses of physical and chemical properties and the proportions prediction of different substrates in mixtures. Therefore, this paper try to establish the prediction model between the mixture proportions and the target properties of the substrate by the parameters analysis of the multiple regression equations, which based on designed the substrate mixed formulation and determination of the physical and chemical properties of the mixed substrate. Furthermore, a comprehensive performance model will be established by weighted summation of the individual property prediction models. The comprehensive performance model will determine the best mixed formulation by genetic algorithm under the condition of setting the target characteristic value. The result will provide a simple and scientific method for property evaluation and formulations design of mixed soilless substrate for agronomic and horticultural production.

\section{Materials and methods}

\subsection{Single-substrate materials and design of mixed-substrate formulations}

This study was conducted in the College of Horticulture as well as Collaborative Innovation Center of Vegetable Industry in Hebei Agricultural University from 2017 to 2019. In this experiment, five kinds of single-substrate materials were collected from commercial substrate company, including vermiculite (Lingshou Lvjin Seedlings Substrate Processing Co., China), peat ${ }^{\mathbb{D}}$ (Liaoning Chuangu Agricultural Technology Co., Ltd., China), coconut coir (Pelemix Ltd., Israel), mushroom residue (Lingshou Lvjin Seedlings Substrate Processing Co., China), and perlite (Lingshou County Haoqian Mineral Powder Processing Factory, China). All the individual substrates were dried in an $80^{\circ} \mathrm{C}$ oven to constant dry mass and prepared to mix. The mixture ratios of the substrates were designed according to the simplex lattice $\operatorname{method}^{[30,31]}$ in Minitab 17. In this method, the five kinds of substrate (vermiculite, peat ${ }^{(\mathbb{1}}$, coconut coir, mushroom residue and perlite) were evaluated as the five main factors by changing their proportions simultaneously and keeping their total proportion across all formulations constant. The ratio of single-substrate factor $X_{i}$ was restricted in accordance with the constraint conditions of Equation (1).

$$
X_{1}+X_{2}+X_{3}+X_{4}+X_{5}=1, \quad 0 \leq X_{i} \leq 1 \quad(i=1,2,3,4,5)
$$

where, $X_{1}, X_{2}, X_{3}, X_{4}$ and $X_{5}$ represent the volume ratios of the individual substrates.

A total of 76 substrate mixtures were formulated employing eight different volume ratios of individual substrates, i.e., 0, 0.1, $0.2,0.25,0.5,0.6,0.75$ and 1 (Table 1). The mixed substrate of every formulation was mixed uniformly according to the volume ratio of individual substrate in the laboratory and kept the amount of each mixed substrate was 1.2 L. Then 76 mixed substrate were divided into 6 equal parts respectively to determine the every value of physical and chemical properties.

Table 1 Formulation numbers and volume ratios (vermiculite: peat ${ }^{\mathbb{D}}$ : coconut coir: mushroom residue: perlite) of the mixed substrates

\begin{tabular}{|c|c|c|c|}
\hline Number & Substrate ratio & Number & Substrate ratio \\
\hline 1 & $0.5: 0: 0: 0.5: 0$ & 39 & $0.1: 0.1: 0.1: 0.1: 0.6$ \\
\hline 2 & $0: 0: 0.25: 0.25: 0.5$ & 40 & $0: 1: 0: 0: 0$ \\
\hline 3 & $0.75: 0: 0: 0.25: 0$ & 41 & $0: 0.5: 0.25: 0.25: 0$ \\
\hline 4 & $0.75: 0: 0: 0: 0.25$ & 42 & $0.25: 0.25: 0: 0.5: 0$ \\
\hline 5 & $0: 0.5: 0: 0.5: 0$ & 43 & $0.25: 0: 0.25: 0.5: 0$ \\
\hline 6 & $0: 0.25: 0: 0: 0.75$ & 44 & $0.25: 0.5: 0: 0.25: 0$ \\
\hline 7 & $0: 0: 0.75: 0.25: 0$ & 45 & $0: 0: 0: 0.25: 0.75$ \\
\hline 8 & $0.25: 0.25: 0: 0: 0.5$ & 46 & $0: 0: 0.5: 0: 0.5$ \\
\hline 9 & $0.25: 0.5: 0.25: 0: 0$ & 47 & $0: 0: 0: 1: 0$ \\
\hline 10 & $0.1: 0.1: 0.1: 0.6: 0.1$ & 48 & $0: 0: 0.25: 0: 0.75$ \\
\hline 11 & $0.5: 0.25: 0: 0.25: 0$ & 49 & $0.1: 0.6: 0.1: 0.1: 0.1$ \\
\hline 12 & $0: 0: 0.75: 0: 0.25$ & 50 & $0.25: 0: 0: 0: 0.75$ \\
\hline 13 & $0: 0.5: 0.25: 0: 0.25$ & 51 & $0.25: 0.25: 0.25: 0: 0.25$ \\
\hline 14 & $0: 0: 0.5: 0.5: 0$ & 52 & $0: 0: 0.25: 0.75: 0$ \\
\hline 15 & $0.25: 0.25: 0: 0.25: 0.25$ & 53 & $0.5: 0: 0: 0.25: 0.25$ \\
\hline 16 & $0.25: 0: 0: 0.5: 0.25$ & 54 & $0: 0.5: 0.5: 0: 0$ \\
\hline 17 & $0: 0.25: 0.25: 0.25: 0.25$ & 55 & $0.5: 0: 0: 0: 0.5$ \\
\hline 18 & $0.25: 0.5: 0: 0: 0.25$ & 56 & $0.2: 0.2: 0.2: 0.2: 0.2$ \\
\hline 19 & $0: 0.25: 0.75: 0: 0$ & 57 & $0: 0: 0: 0.5: 0.5$ \\
\hline 20 & $0: 0.25: 0.25: 0.5: 0$ & 58 & $0.6: 0.1: 0.1: 0.1: 0.1$ \\
\hline 21 & $0: 0.25: 0: 0.75: 0$ & 59 & $0: 0: 0.25: 0.5: 0.25$ \\
\hline 22 & 1:0:0:0:0 & 60 & $0.5: 0: 0.5: 0: 0$ \\
\hline 23 & $0: 0.25: 0: 0.25: 0.5$ & 61 & $0.25: 0: 0: 0.75: 0$ \\
\hline 24 & $0.25: 0: 0.5: 0.25: 0$ & 62 & 0:0:0:0:1 \\
\hline 25 & $0: 0: 1: 0: 0$ & 63 & $0: 0.75: 0.25: 0: 0$ \\
\hline 26 & $0.25: 0.25: 0.25: 0.25: 0$ & 64 & $0.5: 0.25: 0.25: 0: 0$ \\
\hline 27 & $0: 0.25: 0.25: 0: 0.5$ & 65 & $0: 0.5: 0: 0: 0.5$ \\
\hline 28 & $0.5: 0: 0.25: 0.25: 0$ & 66 & $0: 0.25: 0: 0.5: 0.25$ \\
\hline 29 & $0.25: 0.25: 0.5: 0: 0$ & 67 & $0: 0.5: 0: 0.25: 0.25$ \\
\hline 30 & $0.25: 0: 0: 0.25: 0.5$ & 68 & $0.1: 0.1: 0.6: 0.1: 0.1$ \\
\hline 31 & $0.25: 0: 0.25: 0.25: 0.25$ & 69 & $0.25: 0: 0.25: 0: 0.5$ \\
\hline 32 & $0.25: 0: 0.75: 0: 0$ & 70 & $0.25: 0.75: 0: 0: 0$ \\
\hline 33 & $0.5: 0.25: 0: 0: 0.25$ & 71 & $0.75: 0.25: 0: 0: 0$ \\
\hline 34 & $0.5: 0.5: 0: 0: 0$ & 72 & $0: 0: 0: 0.75: 0.25$ \\
\hline 35 & $0.25: 0: 0.5: 0: 0.25$ & 73 & $0.5: 0: 0.25: 0: 0.25$ \\
\hline 36 & $0.75: 0: 0.25: 0: 0$ & 74 & $0: 0.75: 0: 0.25: 0$ \\
\hline 37 & $0: 0.25: 0.5: 0: 0.25$ & 75 & $0: 0.75: 0: 0: 0.25$ \\
\hline 38 & $0: 0.25: 0.5: 0.25: 0$ & 76 & $0: 0: 0.5: 0.25: 0.25$ \\
\hline
\end{tabular}

2.2 Determination of the physical and chemical properties of the mixed substrates

The investigated physical properties of the substrates were the bulk density (DB), total porosity (TP), water-holding porosity (WHP), air porosity (AP), WHP/AP, which were determined using 
loosely packed cores in accordance with the methods of Vaughn et al. ${ }^{[17]}$ and Webber et al. ${ }^{[32]}$ The examined chemical properties included electrical conductivity (EC) and cation exchange capacity (CEC). The value of DB was calculated by dividing the mass ( $\mathrm{g}$ ) of mixed substrate by the volume $\left(\mathrm{cm}^{3}\right)$. The value of AP and WHP, which represent the space occupied by air and water, respectively, in the mixed substrate, were each expressed as percentage (\%) after dividing the volume of pore diameters greater than $0.1 \mathrm{~mm}$ or the volume of pore diameters between 0.001 $0.1 \mathrm{~mm}$ by the mixed substrate volume. The value of TP was calculated as the sum of AP and WHP for each mixed substrate formulation. The value of EC was used to quantify the concentration of soluble salts, which was measured by the filtrate of mixed substrate and water mixed at ratio of 1:5 (v: v) for $1 \mathrm{~h}$ in accordance with the method of Belda et al. ${ }^{[4]}$ using an HI9813 portable EC meter (Hanna Instruments, Woonsocket, RI, USA). The CEC value was determined by the ammonium acetate extraction method at $\mathrm{pH} 7.0$ according to Sparks's method ${ }^{[33]}$. The measurement of each physical and chemical property was replicated six times. Each property listed in the table was the average value of six replicates, which was also used to establish regression equations.

\subsection{Establishment and verification of prediction models for} each physical and chemical property

\subsubsection{Establishment of regression equations}

Regression equations of Equation (2) were established using the ratios of the individual substrates (vermiculite, peat ${ }^{(\mathbb{D}}$, coconut coir, mushroom residue and perlite) as independent variables to evaluate the responses of DB, TP, WHP, AP, WHP/AP, EC and CEC.

$$
\left\{\begin{array}{l}
Y=\left(f_{1}(X), f_{2}(X), f_{3}(X), f_{4}(X), f_{5}(X), f_{6}(X), f_{7}(X),\right)^{\mathrm{T}} \\
f_{i}(X)=\partial_{i 1} f\left(x_{1}\right)+\partial_{i 2} f\left(x_{2}\right)+\partial_{i 3} f\left(x_{3}\right)+\partial_{i 4} f\left(x_{4}\right)+\partial_{i 5} f\left(x_{5}\right)
\end{array}\right.
$$

where, $Y$ is the model of each physical or chemical property of mixed substrate (with $f_{1}(X)$ representing the DB model, $f_{2}(X)$ representing the TP model, $f_{3}(X)$ representing the WHP model, $f_{4}(X)$ representing the AP model, $f_{5}(X)$ representing the WHP/AP model, $f_{6}(X)$ representing the EC model and $f_{7}(X)$ representing the CEC model). $f_{i}(X)$ is a function of mixed substrate, $f\left(x_{1}\right), f\left(x_{2}\right)$, $f\left(x_{3}\right), f\left(x_{4}\right)$ and $f\left(x_{5}\right)$ are the functions of the vermiculite, peat, coconut coir, mushroom residue and perlite composition respectively, and $\partial_{i j}$ is the coefficient of function $(j=1,2,3,4,5)$.

2.3.2 Establishment of prediction models of the individual physical and chemical properties of mixed substrates

Based on the established regression equation for each physical or chemical property, linear equations of all the physical and chemical properties were derived, and it was determined that the values of individual parameters were similar to the coefficients of the linear equations. Seven prediction models of the physical and chemical properties of mixed substrate were constructed according to the association degree, as shown in Equation (3), in which the equation coefficients were replaced with measured property values.

$$
\left\{\begin{array}{l}
Z=\left(Z_{1}(X), Z_{2}(X), Z_{3}(X), Z_{4}(X), Z_{5}(X), Z_{6}(X), Z_{7}(X)\right)^{\mathrm{T}} \\
Z_{i}(X)=\alpha_{i 1} x_{1}+\alpha_{i 2} x_{2}+\alpha_{i 3} x_{3}+\alpha_{i 4} x_{4}+\alpha_{i 5} x_{5}
\end{array}\right.
$$

where, $Z$ is the new model of the physical or chemical properties of the mixed substrates; $Z_{i}(X)$ is the structure of the model, which is a linear equation (with $Z_{1}(X)$ representing the new DB model, $Z_{2}(X)$ representing the new TP model, $Z_{3}(X)$ representing the new WHP model, $Z_{4}(X)$ representing the new AP model, $Z_{5}(X)$ representing the new WHP/AP model, $Z_{6}(X)$ representing the new EC model and $Z_{7}(X)$ representing the new CEC model); and $\alpha_{i j}$ is the value of a single parameter $(j=1,2,3,4,5)$.

2.3.3 Verification of the accuracies of the prediction models of physical and chemical properties

Seven substrates, which were vermiculite (Lingshou Lvjin Seedlings Substrate Processing Co., China), peat ${ }^{(1)}$ (Liaoning Chuangu Agricultural Technology Co., Ltd., China), coconut coir (Pelemix Ltd., Israel), mushroom residue (Lingshou Lvjin Seedlings Substrate Processing Co., China), peat ${ }^{(2)}$ (Pindstrup, Denmark), cassava residue (Huai'an Chaimihe Agriculture Science and Technology Co., Ltd., China) and vinegar residue (Jiangsu Peilei Substrate Technology Development Co., Ltd., China) were combined into 12 different mixed substrates (Table 2) to detect the physical and chemical properties. The mixed substrate of every formulation was mixed uniformly according to the volume ratio of individual substrate and kept the amount of each mixed substrate was $1.2 \mathrm{~L}$. Then it was divided into 6 equal parts respectively to determine the every value of physical and chemical properties. Relative error was used to analyse the degree of conformity between the measured physical and chemical properties of the mixed substrates and the predicted values from the regression equations. The REs of the predicted and measured values of the physical and chemical properties of the newly produced mixed substrates were analysed to verify the stability and accuracy of each physical or chemical property prediction model.

$$
R E=\frac{R_{M S E}}{\text { Sample average }}
$$

\begin{tabular}{|c|c|}
\hline Formulation & Composition of mixed substrate \\
\hline $\mathrm{S} 1$ & Peat $^{(2)}:$ mushroom residue: vermiculite $=2: 6: 2$ \\
\hline $\mathrm{S} 2$ & Peat ${ }^{(2)}:$ mushroom residue: vermiculite $=5: 3: 2$ \\
\hline $\mathrm{S} 3$ & Peat $^{(2)}:$ mushroom residue: vermiculite $=7: 1: 2$ \\
\hline S4 & Vinegar residue: peat $^{(1)}:$ vermiculite $=2: 6: 2$ \\
\hline S5 & Vinegar residue: peat $^{(1)}:$ vermiculite $=5: 3: 2$ \\
\hline S6 & Vinegar residue: peat $^{(1)}:$ vermiculite $=7: 1: 2$ \\
\hline S7 & Cassava: coconut coir: vermiculite $=2: 6: 2$ \\
\hline S8 & Cassava: coconut coir: vermiculite $=5: 3: 2$ \\
\hline S9 & Cassava: coconut coir: vermiculite $=7: 1: 2$ \\
\hline $\mathrm{S} 10$ & Peat $^{(2)}:$ cassava: $^{\text {vinegar residue }}=1: 2: 7$ \\
\hline S11 & Peat $^{(2)}$ : cassava: vinegar residue $=2: 3: 5$ \\
\hline S12 & Peat $^{(2)}$ : cassava: vinegar residue $=4: 4: 2$ \\
\hline
\end{tabular}

Table 2 Mixed-substrate formulations and associated volume ratios used to verify the property prediction models

2.4 Determination of weight coefficients and construction of the comprehensive property model

2.4.1 Construction of the comprehensive property model

The seven independent DB, TP, WHP, AP, WHP/AP, EC and CEC models were combined into a comprehensive property model (Equation (5)) by weighted summation. In this comprehensive property model, the optimal value of each parameter of the substrate had to be set separately, such as $\mathrm{DB}_{0}, \mathrm{TP}_{0}, \mathrm{WHP}_{0}, \mathrm{AP}_{0}$, $\mathrm{WHP}_{0} / \mathrm{AP}_{0}, \mathrm{EC}_{0}$ and $\mathrm{CEC}_{0}$. In the future use of the model, the optimal values can be set according to the needs of the researcher. Refer to the literature ${ }^{[5,7,17]}$ to determine the suitable physical and chemical properties of the substrates: $0.19-0.7 \mathrm{~g} / \mathrm{cm}^{3}$ for $\mathrm{DB}$, $50 \%-85 \%$ for TP, $45 \%-64 \%$ for WHP porosity, $10 \%-30 \%$ for AP, 1:2-4 for WHP/AP, $0.5-1.6 \mathrm{mS} / \mathrm{cm}$ for EC, and $10-100 \mathrm{cmol} / \mathrm{kg}$ for CEC. Accordingly, in this study, $\mathrm{DB}_{0}$ was set to $0.29, \mathrm{TP}_{0}$ was set to $0.79, \mathrm{WHP}_{0}$ was set to $0.59, \mathrm{AP}_{0}$ was set to $0.20, \mathrm{WHP}_{0} / \mathrm{AP}_{0}$ was set to $0.34, \mathrm{EC}_{0}$ was set to 0.84 , and $\mathrm{CEC}_{0}$ was set to 28.35 . The comprehensive property model was then established, which 
can be used to obtain the optimal substrate formulation.

$$
\min Z=\gamma_{1} \min Z_{1}+\gamma_{2} \min Z_{2}+\ldots+\gamma_{7} \min Z_{7}
$$

where, $Z$ is the model equation for the physical or chemical property of the mixed substrate; $Z_{1}$ represents the new DB model; $Z_{2}$ represents the new TP model; $Z_{3}$ represents the new WHP model; $Z_{4}$ represents the new AP model; $Z_{5}$ represents the new WHP/AP model; $Z_{6}$ represents the new EC model; and $Z_{7}$ represents the new CEC model; $\gamma_{i}$ is the weighted coefficient $(i=1,2,3,4,5,6,7)$.

2.4.2 Setting the weight coefficients of the comprehensive property model

The target parameters, such as DB, TP, WHP, AP, WHP/AP, $\mathrm{EC}$ and $\mathrm{CEC}$, were standardized and then subjected to principal component analysis. The principal component with a feature root greater than 1 and a cumulative contribution rate that reached $80 \%$ were selected to establish a weight coefficient (Equation (6)).

$$
\gamma_{i}=\sum_{i=1}^{m} u_{n m} \beta_{i} / k
$$

where, $u_{n m}$ is the coefficient of the decision substrate of the target indicator in each principal component analysis and $k=\beta_{1}+\beta_{2}+\ldots+\beta_{m}$ is the covariance contribution rate of the principal components.

\subsection{Optimal substrate formulations obtained from the comprehensive property model}

Genetic algorithm ${ }^{[34-36]}$ was used to identify the non-inferior solution set of the function as the optimization formulation. The genetic algorithm was used for multi-objective optimization of the comprehensive property model based on the following parameter settings: population size, 20; crossover probability, 0.8 ; mutation probability, 0.2 ; and maximum evolution number, 100 . The substrates used for verification includes vermiculite (Lingshou Lvjin Seedlings Substrate Processing Co., China), coconut coir (Pelemix Ltd., Israel), mushroom residue (Lingshou Lvjin Seedlings Substrate Processing Co., China), peat ${ }^{(2)}$ (Pindstrup, Denmark), perlite (Lingshou County Haoqian Mineral Powder Processing Factory, China), cassava residue (Huai'an Chaimihe Agriculture Science and Technology Co., Ltd., China) and vinegar residue (Jiangsu Peilei Substrate Technology Development Co., Ltd., China). They were mixed according to the optimization formulation, The mixed substrate kept the amount as $1.2 \mathrm{~L}$, then it was divided into 6 equal parts and was determined the physical and chemical properties respectively. The degree of deviation between the measured value and the predicted value was analysed via the RE to test the prediction accuracy of the optimization formulation.

\subsection{Statistical analysis}

The data of physical and chemical properties of mixed substrate were pre-processed by Microsoft Excel 2016. The regression equations of the physical and chemical properties and principal component analysis were calculated and performed, respectively, via SPSS 22.0. The multi-objective optimization analysis was performed using the genetic algorithm tool in the optimization toolbox of MATLAB R2018a, and the search results were statistically analysed using SPSS 22.0 software.

\section{Results}

\subsection{Physical and chemical characteristics of the mixed substrates}

The values of DB, TP, WHP, AP, WHP/AP, EC and CEC significantly differed among the 76 formulations containing 5 substrates in different proportions (vermiculite, peat ${ }^{(1)}$, coconut coir, mushroom residue, perlite) (Table 3). Across the formulations, the values of DB ranged between 0.12 and $0.46 \mathrm{~g} / \mathrm{cm}^{3}$, the values of TP ranged between $56.82 \%$ and $82.05 \%$, the values of WHP ranged from $38.93 \%$ to $71.14 \%$, the values of AP ranged from $4.61 \%$ to $24.51 \%$, the values of EC ranged from 0.02 to $1.65 \mathrm{mS} / \mathrm{cm}$ and the values of CEC ranged from 10 to $61.25 \mathrm{cmol} / \mathrm{kg}$. The formulation comprised only of vermiculite (No.22) had the highest WHP and the lowest AP, EC, and the CEC values among the substrate mixtures. As the proportion of vermiculite in the substrate increased, the values of WHP increased, whereas the values of $\mathrm{AP}, \mathrm{EC}$ and $\mathrm{CEC}$ changed little. The formulation containing only coconut coir (No.25) had the highest TP value and the lowest DB value among the formulations and high WHP value. As the proportion of coconut coir in the formulation increased, the values of TP and WHP rapidly increased, whereas DB values underwent minor changes. The formulation containing only peat ${ }^{\mathbb{D}}$ (No.40) had the highest DB value among the substrate mixtures and higher values of AP, EC, and CEC. As the proportion of peat $^{(1)}$ in the substrate increased, the values of DB, AP, EC, and CEC increased significantly. The formulation containing only mushroom residue (No.47) had the highest EC value among the substrate mixtures and high values of DB, TP, WHP, and CEC. The values of EC, DB, TP, WHP and CEC in the mixed substrate greatly increased as the proportion of mushroom residue increased. The formulation containing only perlite (No.62) had the highest AP value among the formulations and lower values of DB, WHP, EC and CEC. As the proportion of perlite in the substrate increased, AP greatly increased, whereas DB, WHP, EC and CEC did not significantly change.

\begin{tabular}{|c|c|c|c|c|c|c|c|}
\hline Formulation & $\mathrm{DB} / \mathrm{g} \cdot \mathrm{cm}^{-3}$ & $\mathrm{AP} / \%$ & $\mathrm{WHP} / \%$ & $\mathrm{TP} / \%$ & WHP/AP & $\mathrm{EC} / \mathrm{mS} \cdot \mathrm{cm}^{-1}$ & $\mathrm{CEC} / \mathrm{cmol} \cdot \mathrm{kg}^{-1}$ \\
\hline 1 & 0.31 & 12.57 & 61.50 & 74.07 & 0.20 & 0.87 & 39.00 \\
\hline 2 & 0.20 & 19.19 & 49.49 & 68.69 & 0.39 & 0.62 & 29.25 \\
\hline 4 & 0.18 & 9.36 & 65.66 & 75.03 & 0.14 & 0.02 & 12.00 \\
\hline 5 & 0.38 & 17.55 & 57.20 & 74.75 & 0.31 & 1.24 & 47.75 \\
\hline 6 & 0.22 & 12.06 & 44.76 & 56.82 & 0.27 & 0.24 & 20.75 \\
\hline 7 & 0.20 & 17.05 & 60.93 & 77.97 & 0.28 & 0.85 & 37.75 \\
\hline 8 & 0.23 & 9.36 & 52.42 & 61.78 & 0.18 & 0.20 & 15.50 \\
\hline 9 & 0.27 & 10.84 & 60.59 & 71.43 & 0.18 & 0.52 & 23.50 \\
\hline 10 & 0.30 & 18.03 & 62.61 & 80.64 & 0.29 & 1.21 & 39.25 \\
\hline 11 & 0.33 & 11.05 & 61.67 & 72.72 & 0.18 & 0.65 & 24.50 \\
\hline 12 & 0.14 & 13.97 & 61.01 & 74.98 & 0.23 & 0.44 & 20.75 \\
\hline 13 & 0.25 & 13.57 & 53.75 & 67.32 & 0.25 & 0.51 & 23.00 \\
\hline 15 & 0.29 & 10.13 & 57.82 & 67.95 & 0.18 & 0.69 & 33.00 \\
\hline
\end{tabular}

Table 3 Physical and chemical properties of the $\mathbf{7 6}$ mixed-substrate formulations 


\begin{tabular}{|c|c|c|c|c|c|c|c|}
\hline Formulation & $\mathrm{DB} / \mathrm{g} \cdot \mathrm{cm}^{-3}$ & $\mathrm{AP} / \%$ & WHP/\% & $\mathrm{TP} / \%$ & WHP/AP & $\mathrm{EC} / \mathrm{mS} \cdot \mathrm{cm}^{-1}$ & $\mathrm{CEC} / \mathrm{cmol} \cdot \mathrm{kg}^{-1}$ \\
\hline 16 & 0.23 & 14.44 & 54.16 & 68.60 & 0.27 & 0.79 & 28.50 \\
\hline 17 & 0.26 & 19.73 & 54.35 & 74.08 & 0.36 & 0.81 & 29.75 \\
\hline 18 & 0.28 & 13.11 & 54.48 & 67.59 & 0.24 & 0.37 & 20.50 \\
\hline 19 & 0.24 & 12.76 & 63.32 & 76.08 & 0.20 & 0.60 & 30.50 \\
\hline 20 & 0.33 & 19.50 & 58.65 & 78.15 & 0.33 & 1.07 & 39.50 \\
\hline 21 & 0.36 & 20.34 & 56.02 & 76.35 & 0.36 & 1.48 & 54.75 \\
\hline 23 & 0.27 & 20.56 & 48.29 & 68.85 & 0.43 & 0.55 & 21.50 \\
\hline 24 & 0.19 & 10.04 & 63.09 & 73.14 & 0.16 & 0.77 & 25.75 \\
\hline 25 & 0.12 & 11.92 & 67.52 & 79.44 & 0.18 & 0.67 & 21.75 \\
\hline 26 & 0.30 & 14.00 & 61.69 & 75.69 & 0.23 & 0.86 & 33.50 \\
\hline 27 & 0.22 & 12.08 & 51.07 & 63.15 & 0.24 & 0.33 & 20.00 \\
\hline 28 & 0.25 & 9.79 & 61.75 & 71.55 & 0.16 & 0.59 & 20.75 \\
\hline 29 & 0.24 & 8.96 & 63.19 & 72.16 & 0.14 & 0.52 & 20.50 \\
\hline 31 & 0.23 & 11.24 & 60.22 & 71.46 & 0.19 & 0.62 & 29.00 \\
\hline 32 & 0.18 & 6.38 & 69.30 & 75.68 & 0.09 & 0.41 & 21.50 \\
\hline 33 & 0.26 & 10.33 & 56.99 & 67.32 & 0.18 & 0.21 & 16.25 \\
\hline 34 & 0.34 & 11.69 & 58.36 & 70.05 & 0.20 & 0.46 & 22.50 \\
\hline 35 & 0.16 & 8.01 & 61.65 & 69.66 & 0.13 & 0.29 & 27.25 \\
\hline 36 & 0.26 & 4.61 & 69.52 & 74.13 & 0.07 & 0.15 & 11.00 \\
\hline 37 & 0.22 & 14.92 & 48.57 & 63.49 & 0.31 & 0.52 & 19.00 \\
\hline 38 & 0.28 & 13.67 & 63.14 & 76.81 & 0.22 & 0.87 & 29.00 \\
\hline 39 & 0.21 & 10.87 & 54.83 & 65.70 & 0.20 & 0.36 & 17.25 \\
\hline 40 & 0.43 & 17.46 & 57.00 & 74.25 & 0.31 & 0.87 & 27.25 \\
\hline 41 & 0.32 & 17.59 & 58.56 & 76.15 & 0.30 & 0.85 & 30.50 \\
\hline 42 & 0.38 & 19.12 & 62.94 & 82.05 & 0.30 & 1.04 & 25.00 \\
\hline 43 & 0.33 & 18.07 & 63.91 & 81.97 & 0.28 & 1.00 & 38.50 \\
\hline 46 & 0.14 & 12.68 & 50.66 & 63.34 & 0.25 & 0.32 & 21.75 \\
\hline 47 & 0.34 & 22.68 & 53.74 & 76.42 & 0.42 & 1.65 & 54.75 \\
\hline 48 & 0.16 & 13.48 & 49.65 & 63.13 & 0.27 & 0.17 & 14.75 \\
\hline 49 & 0.34 & 16.47 & 57.64 & 74.11 & 0.29 & 0.72 & 27.50 \\
\hline 50 & 0.17 & 14.57 & 50.44 & 65.01 & 0.29 & 0.03 & 14.25 \\
\hline 51 & 0.25 & 7.24 & 60.95 & 68.19 & 0.12 & 0.36 & 18.75 \\
\hline 52 & 0.28 & 24.51 & 54.32 & 78.84 & 0.45 & 1.36 & 53.00 \\
\hline 53 & 0.24 & 9.43 & 57.31 & 66.73 & 0.16 & 0.40 & 24.00 \\
\hline 54 & 0.28 & 15.84 & 59.45 & 75.29 & 0.27 & 0.69 & 34.75 \\
\hline 55 & 0.19 & 7.49 & 58.78 & 66.27 & 0.13 & 0.02 & 23.25 \\
\hline 56 & 0.26 & 9.70 & 58.99 & 68.69 & 0.16 & 0.68 & 28.50 \\
\hline 57 & 0.25 & 19.47 & 50.16 & 69.63 & 0.39 & 0.96 & 51.25 \\
\hline 58 & 0.27 & 7.00 & 66.58 & 73.58 & 0.11 & 0.34 & 18.25 \\
\hline 59 & 0.23 & 20.70 & 51.56 & 72.26 & 0.40 & 1.01 & 34.25 \\
\hline 60 & 0.21 & 8.68 & 69.12 & 77.80 & 0.13 & 0.35 & 26.25 \\
\hline 61 & 0.32 & 19.61 & 58.38 & 77.99 & 0.34 & 1.30 & 53.00 \\
\hline 62 & 0.13 & 23.05 & 38.93 & 61.98 & 0.59 & 0.05 & 10.75 \\
\hline 63 & 0.34 & 17.19 & 58.39 & 75.58 & 0.29 & 0.74 & 28.00 \\
\hline 64 & 0.29 & 8.32 & 61.25 & 69.58 & 0.14 & 0.35 & 19.75 \\
\hline 65 & 0.29 & 12.28 & 50.50 & 62.78 & 0.24 & 0.37 & 18.50 \\
\hline 66 & 0.32 & 20.92 & 51.88 & 72.80 & 0.40 & 0.93 & 36.00 \\
\hline 67 & 0.30 & 11.72 & 52.00 & 63.72 & 0.23 & 0.49 & 28.50 \\
\hline 68 & 0.21 & 13.43 & 67.10 & 80.53 & 0.20 & 0.60 & 26.00 \\
\hline 69 & 0.17 & 8.03 & 51.15 & 59.18 & 0.16 & 0.15 & 12.75 \\
\hline 70 & 0.34 & 16.56 & 57.05 & 73.62 & 0.29 & 0.61 & 19.75 \\
\hline 71 & 0.29 & 7.30 & 68.39 & 75.69 & 0.11 & 0.19 & 14.75 \\
\hline 72 & 0.32 & 20.19 & 52.27 & 72.46 & 0.39 & 1.23 & 61.25 \\
\hline 73 & 0.18 & 7.59 & 60.92 & 68.51 & 0.12 & 0.15 & 12.25 \\
\hline
\end{tabular}

Note: DB: bulk density; TP: total porosity; WHP: water-holding porosity; AP: air porosity; EC: electrical conductivity; CEC: cation exchange capacity. The same abbreviation definitions apply in the tables that follow. 


\subsection{Seven prediction models of the physical and chemical} properties of mixed substrates

\subsubsection{Regression equations of physical and chemical properties}

Since no multi-collinearity was identified among the volume ratios of the individual substrates (as independent variables), regression equations were constructed (Table 4) based on the ratio of each substrate (vermiculite, peat ${ }^{\mathbb{D}}$, coconut coir, mushroom residue and perlite) and used to evaluate the responses of DB, TP, WHP, AP, WHP/AP, EC and CEC. All the regression equations were linear equations and the adjusted correlation coefficient $\left(R_{\text {adj }}^{2}\right)$ values of the regression equations for DB, TP, WHP, AP, WHP/AP, $\mathrm{EC}$ and $\mathrm{CEC}$ were $0.92,0.80,0.85,0.80,0.79,0.97$ and 0.82 , respectively. Analysis of variance applied to the regression equations revealed that all equations were significant at $p<0.0001$. The regression coefficients indicated that these equations could be used to evaluate the contributions of individual substrates to the
DB, TP, WHP, AP, WHP/AP, EC and CEC of a mixed substrate. The factors $X_{2}$ (the volume ratio of peat ${ }^{\oplus}$ ) and $X_{4}$ (the volume ratio of mushroom residue) had more significant effects on the DB of the substrate mixture than did the other factors; factors $X_{3}$ (the volume ratio of coconut coir) and $X_{4}$ (the volume ratio of mushroom residue) had more significant effects on TP than did the other factors; factor $X_{1}$ (the volume ratio of vermiculite) had a more significant effect on WHP than did the other factors; the factors $X_{4}$ (the volume ratio of mushroom residue) and $X_{2}$ (the volume ratio of peat ${ }^{(\mathbb{1})}$ ) had more significant effects on AP than did the other factors; and factor $X_{4}$ (the volume ratio of mushroom residue) had more significant effects on EC and CEC than did the other factors. The results of this analysis were consistent with those of the single-substrate analysis. The coefficients of the correlations between the regression equation factors and the measured values corresponding to individual substrates were greater than 0.999 , indicating high fit.

Table 4 Analysis of variance results for the regression equations for DB, TP, WHP, AP, WHP/AP, EC, and CEC

\begin{tabular}{|c|c|c|c|c|c|c|}
\hline Property & Regression equation & $S S$ & MS & $F$ & $p$-value & $R_{\text {adj. }}^{2}$ \\
\hline DB & $Y_{1}=0.251 X_{1}+0.417 X_{2}+0.146 X_{3}+0.354 X_{4}+0.137 X_{5}$ & 0.339 & 0.085 & 219.07 & 0.0001 & 0.92 \\
\hline $\mathrm{TP}$ & $Y_{2}=73.0 X_{1}+71.79 X_{2}+77.56 X_{3}+79.29 X_{4}+56.29 X_{5}$ & 0.182 & 0.046 & 75.99 & 0.0001 & 0.80 \\
\hline WHP & $Y_{3}=70.19 X_{1}+55.84 X_{2}+65.5 X_{3}+55.6 X_{4}+41.47 X_{5}$ & 0.268 & 0.067 & 110.90 & 0.0001 & 0.85 \\
\hline $\mathrm{AP}$ & $Y_{4}=2.81 X_{1}+15.98 X_{2}+12.06 X_{3}+23.69 X_{4}+14.82 X_{5}$ & 0.125 & 0.031 & 75.35 & 0.0001 & 0.80 \\
\hline WHP/AP & $Y_{5}=0.011 X_{1}+0.279 X_{2}+0.181 X_{3}+0.421 X_{4}+0.349 X_{5}$ & 0.562 & 0.140 & 72.99 & 0.0001 & 0.79 \\
\hline $\mathrm{EC}$ & $Y_{6}=0.044 X_{1}+0.739 X_{2}+0.609 X_{3}+1.660 X_{4}+0.025 X_{5}$ & 9.800 & 2.450 & 727.61 & 0.0001 & 0.97 \\
\hline CEC & $Y_{7}=11.01 X_{1}+25.41 X_{2}+26.13 X_{3}+59.48 X_{4}+14.46 X_{5}$ & 8088.62 & 2022.16 & 85.90 & 0.0001 & 0.82 \\
\hline
\end{tabular}

Note: $X_{1}, X_{2}, X_{3}, X_{4}$ and $X_{5}$ represent the volume ratios of the individual substrates.

3.2.2 Seven prediction models of the physical and chemical properties of mixed substrates

Based on the regression equations, seven prediction models of the physical and chemical properties of substrate mixtures were established using the measured values of the physical and chemical properties of the component substrates as coefficients (Table 5). The sensitivities of the seven models were analysed to assess their reliability. The results showed that the REs of the DB, TP, WHP, $\mathrm{AP}, \mathrm{WHP} / \mathrm{AP}, \mathrm{EC}$ and CEC model values and the predicted values from the regression equations were $3.85 \%, 3.61 \%, 1.63 \%, 12.99 \%$, $12.38 \%, 7.38 \%$ and $10.12 \%$, respectively. These low $R E$ values demonstrated that the deviations between the models and regression equations were small and that the seven models can be used to predict the physical and chemical properties of the substrate mixtures.

Table 5 Relative prediction errors between the simulation value and the predicted value from the regression equation for each physical and chemical property of the substrate mixtures

\begin{tabular}{ccc}
\hline Property & $\begin{array}{c}\text { Model of the physical and chemical properties } \\
\text { of the substrate mixture }\end{array}$ & $R E s$ \\
\hline DB & $Z_{1}=A_{1} X_{1}+A_{2} X_{2}+\ldots+A_{\mathrm{n}} X_{\mathrm{n}}$ & $3.85 \%$ \\
TP & $Z_{2}=B_{1} X_{1}+B_{2} X_{2}+\ldots+B_{\mathrm{n}} X_{\mathrm{n}}$ & $3.61 \%$ \\
WHP & $\mathrm{Z}_{3}=C_{1} X_{1}+C_{2} X_{2}+\ldots+C_{\mathrm{n}} X_{\mathrm{n}}$ & $1.63 \%$ \\
AP & $Z_{4}=D_{1} X_{1}+D_{2} X_{2}+\ldots+D_{\mathrm{n}} X_{\mathrm{n}}$ & $12.99 \%$ \\
WHP/AP & $Z_{5}=E_{1} X_{1}+E_{2} X_{2}+\ldots+E_{\mathrm{n}} X_{\mathrm{n}}$ & $12.38 \%$ \\
EC & $Z_{6}=F_{1} X_{1}+F_{2} X_{2}+\ldots+F_{\mathrm{n}} X_{\mathrm{n}}$ & $7.38 \%$ \\
CEC & $Z_{7}=G_{1} X_{1}+G_{2} X_{2}+\ldots+G_{\mathrm{n}} X_{\mathrm{n}}$ & $10.12 \%$ \\
\hline
\end{tabular}

Note: $A_{i}, B_{i}, C_{i}, D_{i}, E_{i}, F_{i}$, and $G_{i}$ represent weight ratios for DB, TP, WHP, AP, WHP/AP, EC, and CEC of the component substrate, respectively. $X_{i}$ represent volume ratio of individual component substrate, respectively. REs: relative prediction errors.

3.2.3 Verification of the seven prediction models of the physical and chemical properties of mixed-substrate formulations

The seven prediction models of physical and chemical properties were verified using 12 substrate mixtures made from 7 substrates: peat ${ }^{(\mathbb{D}}$, cassava residue, vinegar residue, peat ${ }^{(2)}$, coconut coir, mushroom residue and vermiculite. Based on the determinations of the physical and chemical properties of the 7 component substrates, the values of the physical and chemical properties of the 12 mixtures were predicted using the DB, TP, WHP, AP, WHP/AP, EC and CEC prediction models (Table 6). The REs of DB, TP, WHP, AP, WHP/AP, EC and CEC obtained by comparing the predicted and measured values of the 12 mixtures were $7.12 \%, 2.81 \%, 2.20 \%, 6.36 \%, 7.51 \%, 9.35 \%$ and $7.52 \%$, respectively. These REs indicated small deviations between the simulated and measured values, confirming that the seven models were reliable and effective.

\subsection{Weight coefficients and establishment of the comprehensive} property model

3.3.1 Weight coefficients of the comprehensive property model

Compared with the seven prediction models of DB, TP, WHP, $\mathrm{AP}, \mathrm{WHP} / \mathrm{AP}, \mathrm{EC}$ and $\mathrm{CEC}$, the comprehensive property model was more useful for finding the optimal mixed substrate formulation. The weight coefficient of each physical and chemical property in the substrate mixtures were obtained by principal component analysis for DB, WHP, AP, WHP/AP, EC and $\mathrm{CEC}$ in the substrate mixtures. The characteristic roots of principal components 1 and 2 were greater than 1 , and their cumulative variance contribution rate reached $82.76 \%$, as shown in Table 7. Therefore, the seven physical and chemical properties of DB, TP, WHP, AP, WHP/AP, EC and CEC could be divided into two principal components which could explain the differences between substrates. Table 8 presents the linear combination coefficients of the physical and chemical properties and their combination coefficients for principal components 1 and 2. The weight coefficients of DB, TP, WHP, AP, WHP/AP, EC and CEC were calculated as $0.1501,0.1936,0.0854,0.1197,0.0657,0.2035$, and 0.1821 , respectively (Table 8 ). 
Table 6 Verification of the seven models by comparing the predicted and measured values of individual substrates and mixed-substrate formulations

\begin{tabular}{|c|c|c|c|c|c|c|c|c|}
\hline \multicolumn{2}{|c|}{ Substrate/formulation } & \multirow{2}{*}{$\frac{\mathrm{DB} / \mathrm{g} \cdot \mathrm{cm}^{-3}}{0.47}$} & \multirow{2}{*}{$\begin{array}{l}\mathrm{TP} / \% \\
68.87\end{array}$} & \multirow{2}{*}{$\begin{array}{c}\mathrm{WHP} / \% \\
58.56\end{array}$} & \multirow{2}{*}{$\begin{array}{c}\mathrm{AP} / \% \\
10.31\end{array}$} & \multirow{2}{*}{$\begin{array}{c}\mathrm{WHP} / \mathrm{AP} \\
0.18\end{array}$} & \multirow{2}{*}{$\frac{\mathrm{EC} / \mathrm{mS} \cdot \mathrm{cm}^{-1}}{0.28}$} & \multirow{2}{*}{$\frac{\mathrm{CEC} / \mathrm{cmol} \cdot \mathrm{kg}^{-1}}{17.75}$} \\
\hline \multirow{6}{*}{$\begin{array}{l}\text { Single } \\
\text { substrate }\end{array}$} & peat $^{(1)}$ & & & & & & & \\
\hline & Cassava & 0.65 & 65.93 & 54.50 & 11.43 & 0.21 & 1.30 & 42.50 \\
\hline & Vinegar residue & 0.33 & 81.69 & 55.79 & 25.90 & 0.46 & 1.61 & 34.50 \\
\hline & Coconut coir & 0.21 & 79.78 & 58.52 & 21.26 & 0.36 & 0.13 & 16.25 \\
\hline & Mushroom residue & 0.26 & 80.42 & 52.79 & 27.63 & 0.52 & 1.08 & 36.75 \\
\hline & Vermiculite & 0.24 & 80.06 & 70.16 & 9.90 & 0.14 & 0.01 & 9.50 \\
\hline \multirow{11}{*}{$\begin{array}{l}\text { Predicted } \\
\text { value }\end{array}$} & $\mathrm{S} 1$ & 0.23 & 81.52 & 58.82 & 22.70 & 0.41 & 0.70 & 31.05 \\
\hline & S2 & 0.19 & 83.28 & 62.65 & 20.63 & 0.34 & 0.45 & 30.68 \\
\hline & $\mathrm{S} 3$ & 0.17 & 84.45 & 65.21 & 19.24 & 0.30 & 0.29 & 30.43 \\
\hline & $\mathrm{S} 4$ & 0.40 & 73.67 & 60.33 & 13.35 & 0.23 & 0.49 & 19.45 \\
\hline & S5 & 0.35 & 77.52 & 59.50 & 18.02 & 0.31 & 0.89 & 24.48 \\
\hline & S7 & 0.31 & 77.07 & 60.05 & 17.02 & 0.29 & 0.34 & 20.15 \\
\hline & S8 & 0.44 & 72.91 & 58.84 & 14.07 & 0.24 & 0.69 & 28.03 \\
\hline & S9 & 0.52 & 70.14 & 58.04 & 12.11 & 0.21 & 0.93 & 33.28 \\
\hline & S10 & 0.37 & 79.00 & 56.51 & 22.49 & 0.40 & 1.41 & 36.20 \\
\hline & S11 & 0.39 & 77.88 & 57.36 & 20.52 & 0.36 & 1.25 & 37.10 \\
\hline & S12 & 0.38 & 77.22 & 59.18 & 18.04 & 0.30 & 0.95 & 38.10 \\
\hline \multirow{10}{*}{$\begin{array}{l}\text { Measured } \\
\text { value }\end{array}$} & $\mathrm{S} 1$ & 0.27 & 87.11 & 63.59 & 23.52 & 0.37 & 0.80 & 28.75 \\
\hline & $\mathrm{S} 2$ & 0.24 & 81.88 & 60.80 & 21.08 & 0.35 & 0.51 & 35.25 \\
\hline & $\mathrm{S} 3$ & 0.19 & 86.63 & 66.18 & 20.45 & 0.31 & 0.36 & 30.25 \\
\hline & $\mathrm{S} 4$ & 0.39 & 75.68 & 62.29 & 13.39 & 0.22 & 0.56 & 21.25 \\
\hline & S5 & 0.32 & 77.91 & 59.79 & 18.12 & 0.30 & 0.84 & 22.25 \\
\hline & S6 & 0.33 & 76.29 & 57.54 & 18.75 & 0.33 & 1.15 & 26.75 \\
\hline & S9 & 0.50 & 71.37 & 60.14 & 11.23 & 0.19 & 0.96 & 36.00 \\
\hline & $\mathrm{S} 10$ & 0.39 & 80.33 & 57.37 & 22.96 & 0.40 & 1.52 & 38.00 \\
\hline & S11 & 0.36 & 81.38 & 57.67 & 23.71 & 0.41 & 1.10 & 37.50 \\
\hline & S12 & 0.40 & 74.43 & 57.96 & 16.47 & 0.28 & 0.85 & 36.25 \\
\hline \multirow{13}{*}{$\begin{array}{l}\text { Relative } \\
\text { prediction error }\end{array}$} & S1 & $15.30 \%$ & $6.42 \%$ & $7.49 \%$ & $3.50 \%$ & $9.55 \%$ & $12.00 \%$ & $8.00 \%$ \\
\hline & S2 & $17.73 \%$ & $1.71 \%$ & $3.05 \%$ & $2.15 \%$ & $1.05 \%$ & $10.40 \%$ & $12.98 \%$ \\
\hline & $\mathrm{S} 3$ & $8.37 \%$ & $2.51 \%$ & $1.47 \%$ & $5.88 \%$ & $2.31 \%$ & $19.00 \%$ & $0.58 \%$ \\
\hline & $\mathrm{S} 4$ & $2.72 \%$ & $2.65 \%$ & $3.14 \%$ & $0.33 \%$ & $5.43 \%$ & $12.16 \%$ & $8.47 \%$ \\
\hline & S5 & $10.41 \%$ & $0.50 \%$ & $0.49 \%$ & $0.52 \%$ & $3.33 \%$ & $5.72 \%$ & $10.00 \%$ \\
\hline & S6 & $0.91 \%$ & $4.98 \%$ & $2.44 \%$ & $12.77 \%$ & $13.80 \%$ & $0.29 \%$ & $4.02 \%$ \\
\hline & S7 & $8.10 \%$ & $1.89 \%$ & $0.41 \%$ & $10.94 \%$ & $13.17 \%$ & $4.56 \%$ & $16.04 \%$ \\
\hline & S8 & $0.42 \%$ & $1.59 \%$ & $0.29 \%$ & $7.37 \%$ & $8.35 \%$ & $13.25 \%$ & $11.73 \%$ \\
\hline & S9 & $4.46 \%$ & $1.72 \%$ & $3.49 \%$ & $7.81 \%$ & $13.09 \%$ & $3.17 \%$ & $7.57 \%$ \\
\hline & $\mathrm{S} 10$ & $4.98 \%$ & $1.66 \%$ & $1.51 \%$ & $2.04 \%$ & $0.48 \%$ & $7.28 \%$ & $4.74 \%$ \\
\hline & S11 & $6.28 \%$ & $4.30 \%$ & $0.54 \%$ & $13.44 \%$ & $12.91 \%$ & $13.65 \%$ & $1.07 \%$ \\
\hline & $\mathrm{S} 12$ & $5.72 \%$ & $3.76 \%$ & $2.12 \%$ & $9.52 \%$ & $6.60 \%$ & $10.67 \%$ & $5.10 \%$ \\
\hline & $\mathrm{RE}$ & $7.12 \%$ & $2.81 \%$ & $2.20 \%$ & $6.36 \%$ & $7.51 \%$ & $9.35 \%$ & $7.52 \%$ \\
\hline
\end{tabular}

Table 7 Principal component analysis of the physical and chemical properties of substrate mixtures

\begin{tabular}{cccc}
\hline Principal component number & Eigen value & Variance contribution rate $/ \%$ & $\mathbf{5 1 . 6 3 0}$ \\
$\mathbf{1}$ & $\mathbf{3 . 6 1 4}$ & $\mathbf{3 1 . 1 3 6}$ & $\mathbf{5 1 . 6 3 0}$ \\
$\mathbf{2}$ & $\mathbf{2 . 1 8 0}$ & 9.972 \\
9 & 0.698 & 6.084 \\
4 & 0.426 & 0.982 & 92.738 \\
5 & 0.069 & 0.154 & 98.821 \\
6 & 0.011 & 0.042 & 99.903 \\
\hline
\end{tabular}


Table 8 Linear combination coefficients of principal components and property weights

\begin{tabular}{cccc}
\hline \multirow{2}{*}{ Property } & \multicolumn{2}{c}{ Linear combination coefficient } & Weight \\
\cline { 2 - 3 } & PC1 & PC2 & \\
\hline DB & 0.2877 & 0.2483 & 0.1501 \\
TP & 0.2388 & 0.5397 & 0.1936 \\
WHP & -0.1390 & 0.6433 & 0.0854 \\
AP & 0.4768 & -0.2123 & 0.1197 \\
WHP/AP & 0.4164 & -0.3727 & 0.0657 \\
EC & 0.4824 & 0.1837 & 0.2035 \\
CEC & 0.4554 & 0.1249 & 0.1821 \\
\hline
\end{tabular}

3.3.2 Establishment of the comprehensive property model

By weighted summation, the seven prediction models of DB, TP, WHP, AP, WHP/AP, EC and CEC were combined into a comprehensive property model. According to the set values of the physical and chemical properties, namely, $\mathrm{DB}_{0}, \mathrm{TP}_{0}, \mathrm{WHP}_{0}, \mathrm{AP}_{0}$, $\mathrm{WHP}_{0} / \mathrm{AP}_{0}, \mathrm{EC}_{0}$ and $\mathrm{CEC}_{0}$, the comprehensive property prediction model was obtained based on the weight coefficients using Equation (7).

$$
\begin{aligned}
\min Z=\gamma_{1} \min Z_{1}+\gamma_{2} \min Z_{2}+L+\gamma_{7} \min Z_{7} \\
=\gamma_{1} \times \sqrt{\left(D b_{0}-Z_{1}\right)^{2}}+\gamma_{2} \times \sqrt{\left(T P_{0}-Z_{2}\right)^{2}}+\gamma_{3} \times \sqrt{\left(W H P_{0}-Z_{3}\right)^{2}}+ \\
\gamma_{4} \times \sqrt{\left(A P_{0}-Z_{4}\right)^{2}}+\gamma_{5} \times \sqrt{\left(\frac{W H P_{0}}{A P_{0}}-Z_{5}\right)^{2}}+\gamma_{6} \times \sqrt{\left(E C_{0}-Z_{6}\right)^{2}}+ \\
\quad 10^{-2} \times \gamma_{7} \times \sqrt{\left(C E C_{0}-Z_{7}\right)^{2}}
\end{aligned}
$$

where, $Z$ is the model equation for the physical or chemical property of the substrate mixture; $Z_{1}$ represents the new DB model; $Z_{2}$ represents the new TP model; $Z_{3}$ represents the new WHP model; $Z_{4}$ represents the new AP model; $Z_{5}$ represents the new WHP/AP model; $Z_{6}$ represents the new EC model; and $Z_{7}$ represents the new CEC model.

\subsection{Determination of the optimal mixed-substrate formulation} by the comprehensive property model

3.4.1 Setting the optimal initial values of physical and chemical properties for mixed substrate

To predict the optimal mixed-substrate formulation, the optimal values of the physical and chemical properties must be set in advance. In the present study, the initial values of $\mathrm{DB}, \mathrm{TP}$, WHP, AP, WHP/AP, EC and CEC were set to 0.29, 0.79, 0.59, $0.20,0.34,0.84$, and 28.35 , respectively; these values are commonly used for vegetable seedlings. The comprehensive property model was thus established as Equation (8).

$$
\begin{aligned}
\min Z= & 0.1501 \times \sqrt{\left(0.29-Z_{1}\right)^{2}}+0.1936 \times \sqrt{\left(0.79-Z_{2}\right)^{2}}+ \\
& 0.0854 \times \sqrt{\left(0.59-Z_{3}\right)^{2}}+0.1197 \times \sqrt{\left(0.20-Z_{4}\right)^{2}}+ \\
& 0.0657 \times \sqrt{\left(0.34-Z_{5}\right)^{2}}+0.2035 \times \sqrt{\left(0.84-Z_{6}\right)^{2}}+ \\
& 10^{-2} \times 0.1821 \times \sqrt{\left(28.35-Z_{7}\right)^{2}}
\end{aligned}
$$

\subsubsection{Genetic algorithm-based multi-objective optimization}

Using the initial values of DB, TP, WHP, AP, WHP/AP, EC and CEC in Equation (9), six kinds of optimization results were obtained, which are shown in Table 9. The compounding agreeability values of the six optimization results were all greater than 0.9 , which indicated that the optimization results are acceptable. Formulations 3, 4, 5 and 6 represent new formulations containing peat ${ }^{2}$, cassava residue and vinegar residue, demonstrating that the multi-objective optimization process can select substrates according to requirements and local conditions. Formulation 2, containing peat ${ }^{2}$, mushroom residue and perlite at a ratio of 2:7:1 and formulation 5, containing vermiculite, mushroom residue, and cassava at a ratio of 3:5:2, achieved results highly matching the set objective, with compounding agreeability values of 0.98 and 0.95 . These values indicated that the predicted parameters well matched the expected values.

3.4.3 Verification of the multi-objective optimization results

The physical and chemical properties of the mixed-substrate formulations were determined according to the six optimization formulations (Table 10). Regarding the seven properties (DB, TP,

\begin{tabular}{|c|c|c|c|c|c|c|c|c|}
\hline \multirow{2}{*}{ Formulation } & \multicolumn{7}{|c|}{ Predicted parameters } & \multirow{2}{*}{$\begin{array}{c}\text { Compounding } \\
\text { agreeability }\end{array}$} \\
\hline & $\mathrm{DB} / \mathrm{g} \cdot \mathrm{cm}^{-3}$ & $\mathrm{TP} / \%$ & $\mathrm{WHP} / \%$ & $\mathrm{AP} / \%$ & WHP/AP & $\mathrm{EC} / \mathrm{mS} \cdot \mathrm{cm}^{-1}$ & $\mathrm{CEC} / \mathrm{cmol} \cdot \mathrm{kg}^{-1}$ & \\
\hline $\mathrm{P} 1$ & 0.27 & 76.99 & 55.43 & 21.56 & 0.40 & 0.84 & 28.39 & 0.94 \\
\hline P2 & 0.29 & 76.29 & 52.65 & 23.62 & 0.46 & 0.81 & 30.35 & 0.98 \\
\hline P3 & 0.28 & 80.86 & 59.95 & 20.91 & 0.35 & 0.84 & 23.73 & 0.93 \\
\hline $\mathrm{P} 4$ & 0.23 & 83.27 & 60.33 & 22.94 & 0.38 & 0.84 & 27.25 & 0.92 \\
\hline P5 & 0.33 & 76.43 & 57.84 & 19.07 & 0.37 & 0.84 & 30.73 & 0.95 \\
\hline P6 & 0.31 & 79.26 & 61.37 & 17.8 & 0.31 & 0.84 & 23.04 & 0.93 \\
\hline
\end{tabular}
WHP, AP, WHP/AP, EC and CEC) of the substrate mixtures, formulation 5 yielded the lowest RE value of $5.94 \%$, and formulation 1 yielded the highest value of $11.38 \%$. The deviations between the predicted and measured values for the

\begin{tabular}{|c|c|c|c|c|c|c|c|c|}
\hline Formulation & $\mathrm{DB} / \mathrm{g} \cdot \mathrm{cm}^{-3}$ & $\mathrm{TP} / \%$ & WHP/\% & $\mathrm{AP} / \%$ & WHP/AP & $\mathrm{EC} / \mathrm{mS} \cdot \mathrm{cm}^{-1}$ & $\mathrm{CEC} / \mathrm{cmol} \cdot \mathrm{kg}^{-1}$ & $\mathrm{RE} / \%$ \\
\hline P1 & 0.31 & 88.43 & 64.31 & 24.13 & 0.38 & 1.015 & 29.75 & 11.38 \\
\hline P2 & 0.34 & 78.43 & 56.6 & 21.83 & 0.39 & 0.974 & 35.25 & 10.68 \\
\hline P3 & 0.26 & 76.72 & 56.93 & 19.79 & 0.35 & 0.903 & 28.75 & 7.27 \\
\hline P4 & 0.19 & 84.22 & 58.58 & 25.64 & 0.44 & 0.801 & 30.25 & 8.91 \\
\hline P5 & 0.37 & 77.19 & 57.99 & 19.21 & 0.33 & 0.914 & 33.5 & 5.94 \\
\hline
\end{tabular}
optimization formulations of the substrate mixtures were small, which indicated that the optimization results were reliable.

Table 9 Multi-objective optimization for target parameter prediction

Note: P1: Vermiculite: peat ${ }^{(2)}:$ mushroom residue $=2: 1: 7 ;$ P2: peat ${ }^{(2)}:$ mushroom residue: perlite $=2: 7: 1 ;$ P3: Vermiculite: coconut coir: vinegar residue $=2: 3: 5 ;$ P4: Coconut

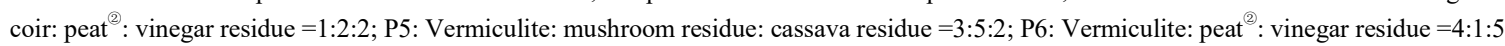

Table 10 Measured values of the physical and chemical properties of substrate mixtures and relative prediction errors 


\section{Discussion}

In a previous study, approximately 50-60 different types of substrates produced in different regions were blended together in different proportions to create mixed substrates close to optimal in terms of meeting plant growth requirements ${ }^{[10]}$. Many researchers have attempted to identify suitable substrate formulations for different crops by conducting small-scale experiments with single substrates at different ratios in the greenhouse or field. Ren et al. ${ }^{[37]}$ reported that a suitable substrate for the growth of tomato seedlings was a formulation of coconut coir and perlite at a ratio of 2:3 (v:v). Yang et al. ${ }^{[38]}$ reported that the growth of Capsicum frutescens L. var. shuanlaense seedlings was the best when the seedlings were grown in mixed substrate containing vegetable garden soil, perlite, vermiculite, and nutrient soil at a volume ratio of 1:2:2:5. Palencia et al. ${ }^{[15]}$ found that strawberry plants grown on agro-textile-type substrate produced significantly more fruit than those grown in other substrates. The above results indicate that different crops have different requirements in terms of the physical and chemical properties of a substrate, and the same crop may benefit from different substrate formulations at different growth stages. Therefore, it is impossible to identify the most suitable substrate for a crop through experimental screening of large numbers of mixed substrates. According to the research, it was expected to build a model that can predict the physical and chemical properties of the mixed substrate based on the physical and chemical properties of a single material to replace the cumbersome formulation test. Firstly, the regression equations between the physical and chemical properties of the mixed substrate (DB, TP, WHP, AP, WHP/AP, EC and CEC) and the ratio of its constituent substrates were constructed by the method of mixture design (Table 1), which has a wide range of applications in food ingredient optimization ${ }^{[39-41]}$. All the regression equations were linear equations, and the high correlation coefficient values for $Y_{1}\left(R_{\text {adj }}^{2}=0.92\right), Y_{2}\left(R_{\text {adj }}^{2}=0.80\right), Y_{3}\left(R_{\text {adj }}^{2}=0.85\right), Y_{4}\left(R_{\text {adj }}^{2}=0.80\right)$, $Y_{5}\left(R_{\text {adj }}^{2}=0.79\right), Y_{6}\left(R_{\text {adj }}^{2}=0.97\right)$ and $Y_{7}\left(R_{\text {adj }}^{2}=0.82\right)$ indicated good fit. The regression equations were all significant $(p<0.0001)$ (Table 4).

The regression model could explain the relationship between the variable and the response well. Juárez-Maldonado et al. ${ }^{[23]}$ used multiple regression models to calculate greenhouse tomato daily evapotranspiration and transpiration rates. Retamales et al. ${ }^{[29]}$ built a regression model to determine the variables that had the greatest influence on blueberry yield. Ulissi et al. ${ }^{[28]}$ compared the spectral reflectance values of leaf and $\mathrm{N}-\mathrm{NO}_{3}$ concentration chemical value by partial least squares chemometric multivariate methods to evaluate the possibility and the accuracy of the estimation of tomato leaf nitrogen concentration performed. However, the variables of the regression model were usually fixed in these studies, and the equation could not be used when the variable changed. According to analysis on the values between coefficients of the regression model and the physical and chemical properties of a single substrate, seven mixed substrate single-property prediction models were constructed with coefficients that can change with the addition of substrate. The prediction models for the individual properties were as follows:

DB, $Z_{1}=A_{1} X_{1}+A_{2} X_{2}+\ldots+A_{n} X_{n} ; \mathrm{TP}, Z_{2}=B_{1} X_{1}+B_{2} X_{2}+\ldots+$ $B_{n} X_{n}$; WHP, $Z_{3}=C_{1} X_{1}+C_{2} X_{2}+\ldots+C_{n} X_{n}$; AP, $Z_{4}=D_{1} X_{1}+D_{2} X_{2}$ $+\ldots+D_{n} X_{n}$; WHP/AP, $Z 5=E_{1} X_{1}+E 2 X_{2}+\ldots+E_{n} X_{n}$; EC, $Z_{6}=$ $F_{1} X_{1}+F_{2} X_{2}+\ldots+F_{n} X_{n} ;$ CEC, $Z_{7}=G_{7} X_{1}+G_{2} X_{2}+\ldots+G_{\mathrm{n}} X_{\mathrm{n}}$
Twelve substrate formulations (Table 2) composed of seven individual substrates were used to validate the models. The REs for DB, TP, WHP, AP, WHP/AP, EC and CEC were 7.12\%, 2.81\%, $2.20 \%, 6.36 \%, 7.51 \%, 9.35 \%$ and $7.52 \%$, respectively, validating the models (Table 6).

To create a single, more broadly applicable model of physical and chemical properties, the seven physical and chemical property models were constructed as a comprehensive property model through linear programming in genetic algorithm, which was an effective method to solve multi-objective optimization. Thangadurai et al. ${ }^{[42]}$ used genetic algorithm to find out the optimal solution for Citrus canker disease identification and solution. Mansini et al. ${ }^{[43]}$ reviewed various portfolio optimization models that can be solved by linear programming. Kanagaraj et al. ${ }^{[4]}$ developed a hybrid cuckoo search algorithm to solve reliability-redundancy optimization problems and global optimization problems, respectively. This model can be used to calculate optimal mixed substrate formulations based on the set-optimal values of the properties of mixed substrate. In this study, the weight coefficient of each physical and chemical property in the mixed substrate was obtained by the principal component analysis (Table 7, Table 8). $\mathrm{DB}_{0}$ was set to $0.29, \mathrm{TP}_{0}$ was set to $0.79, \mathrm{WHP}_{0}$ was set to $0.59, \mathrm{AP}_{0}$ was set to 0.20 , $\mathrm{WHP}_{0} / \mathrm{AP}_{0}$ was set to $0.34, \mathrm{EC}_{0}$ was set to 0.84 , and $\mathrm{CEC}_{0}$ was set to 28.35.The comprehensive performance model was constructed by genetic algorithm, and six highly matched substrate formulations and their predicted values of physical and chemical properties were obtained (Table 9). RE analysis of the predicted and measured values of the six substrate formulations (Table 10) was conducted. The REs between predicted and measured values for the six substrate formulations were $11.38 \%, 10.68 \%, 7.27 \%$, $8.91 \%, 5.94 \%$ and $6.21 \%$, respectively. In practical applications, the expected values of the physical and chemical properties of substrate mixture should be selected according to the cultivation season and crop. The results of this study provide a simple and scientific method for predicting the properties of substrate and for formulating soilless substrates. This study provides important insights that can aid the production and utilization of optimal mixed substrate by researchers and farmers for agronomic and horticultural crop cultivation.

\section{Conclusions}

Seven prediction models of physical and chemical properties (DB, TP, WHP, AP, WHP/AP, EC and CEC) of mixed substrate were constructed based on the regression equations of the determination values of 76 substrate combinations. The models were verified using the measured values from 12 mixed-substrate formulations. A comprehensive property model was established by weighted summing of the seven models of physical and chemical properties and was verified using the measured values of 6 mixed-substrate formulations. Constructed with a large amount of data, the obtained prediction model could effectively predict the physical and chemical properties of mixed substrate and the mixture proportions.

\section{Acknowledgements}

This research was performed at the Collaborative Innovation Center of Vegetable Industry in Hebei, China and was financially supported by the Key Research and Develop Program of Hebei (Grant No. 19227214D, 20326902D). 


\section{[References]}

[1] Barrett G E, Alexander P D, Robinson J S, Bragg N C. Achieving environmentally sustainable growing media for soilless plant cultivation systems - a review. Scientia Horticulturae, 2016; 212: 220-234.

[2] Deepagoda T K K C, Lopez J C C. Integral parameters for characterizing water, energy, and aeration properties of soilless plant growth media. Journal of Hydrology, 2013; 502: 120-127.

[3] Schmilewski G. Growing media constituents in the EU. Acta Hortic, 2009; 819: 33-45.

[4] Belda R M, Lidón A, Fornes F. Biochars and hydrochars as substrate constituents for soilless growth of myrtle and mastic. Industrial Crops and Products, 2016; 94: 132-142.

[5] Bilderback T E, Warren S L, Owen Jr J S, Albano J P. Healthy substrates need physicals too! Hort Technology, 2005; 15: 747-751.

[6] Nelson P V. Greenhouse Operation \& Management, 7th ed. Prentice Hall, Upper Saddle River, NJ, 2011; pp. 607.

[7] Vaughn S F, Kenar J A, Thompson A R. Comparison of biochars derived from wood pellets and pelletized wheat straw as replacements for peat in potting substrates. Industrial Crops and Products, 2013; 51: 437-443.

[8] Meng X M. Problems and counter measures of matrix industry in China. Chinese vegetables, 2017; 8: 16-20. (in Chinese)

[9] Wisdom B, Nyembezi M, Agathar K. Effect of different vermiculite and pine bark media substrates mixtures on physical properties and spiral rooting of radish (Raphanus sativus L.) in float tray system. Rhizosphere, 2017; 3: 67-74.

[10] Mazarura U. Exploring the influence of coal rubble and pine bark substrate mixes on germination, spiral rooting, substrate chemical and physical properties: Using tobacco as test crop. Asian Journal of Agriculture \&Rural Development, 2013; 3: 115-126.

[11] Álvarez, José M, Claudio P, Rattan L. Morpho-physiological plant quality when biochar and vermicompost are used as growing media replacement in urban horticulture. Urban Forestry \& Urban Greening, 2018; 34: 175-180.

[12] Kuisma E, Palonen P, Yli-Halla M. Reed canary grass straw as a substrate in soilless cultivation of strawberry. Scientia Horticulturae, 2014; 178: 217-223.

[13] Lei W S, Ding Y F, Li G H. Effects of soilless substrates on seedling quality and the growth of transplanted super japonica rice. Journal of Agricultural Sciences, 2017; 16: 1053-1063.

[14] Belda R M, Lidón A, Fornes F. Biochars and hydrochars as substrate constituents for soilless growth of myrtle and mastic. Industrial Crops and Products, 2016; 94: 132-142.

[15] Palencia P, Bordonaba J G, Martínez F. Investigating the effect of different soilless substrates on strawberry productivity and fruit composition. Scientia Horticulturae, 2016; 203: 12-19.

[16] Fátima M, Castillo S, Borrero C. Effect of different soilless growing systems on the biological properties of growth media in strawberry. Scientia Horticulturae, 2013; 150: 59-64.

[17] Vaughn S F, Eller F J, Evangelista R L. Evaluation of biochar-anaerobic potato digestate mixtures as renewable components of horticultural potting media. Industrial Crops and Products, 2015; 65: 467-471.

[18] Huanga L, Genhua N. Evaluation of a hardwood biochar and two composts mixes as replacements for a peat-based commercial substrate. Industrial Crops and Products, 2019; 129: 549-560.

[19] Depardieu C. High productivity of soilless strawberry cultivation under rain shelters. Scientia Horticulturae, 2018; 232: 127-138.

[20] Vandecasteele B, Debode J, Willekens K. Recycling of P and K in circular horticulture through compost application in sustainable growing media for fertigated strawberry cultivation. European Journal of Agronomy, 2018; 96: 131-145.

[21] Bouchaaba Z, Santamaria P, Choukrallah R. Open-cycle drip vs closed-cycle subirrigation: Effects on growth and yield of greenhouse soilless green bean. Scientia Horticulturae, 2015; 182: 77-85.

[22] Rubio A J S, Franch V, López F. Towards a near-soilless culture for woody perennial crops in open field conditions. Scientia Horticulturae, 2018; 240: 460-467.

[23] Maldonado A J, Mendoza A B, Romenus K D A. Estimation of the water requirements of greenhouse tomato crop using multiple regression models. Emirates Journal of Food and Agriculture, 2014; 10: 885-897.
[24] Norrie J, Graham M E D, Gosselin A. Potential evapotranspiration as a means of predicting irrigation timing in greenhouse tomatoes grown in peat bags. Journal of the American Society for Horticultural Science, 1994; 119: 163-168.

[25] Ta T H, Shin J H, Ahn T I. Modeling of transpiration of paprika (Capsicum annuum, L.) plants based on radiation and leaf area index in soilless culture. Horticulture Environment and Biotechnology, 2011; 3: 265-269.

[26] Wang Y, Wang F, Huang J. Validation of artificial neural network techniques in the estimation of nitrogen concentration in rape using canopy hyperspectral reflectance data. International Journal of Remote Sensing, 2009; 17: 4493-4505.

[27] Hansen P M, Schjoerring J K. Reflectance Measurement of Canopy Biomass and Nitrogen Status in Wheat Crops Using Normalized Difference Vegetation Indices and Partial Least Squares Regression. Remote Sensing of Environment, 2003; 4: 542-553.

[28] Ulissi V. Nitrogen Concentration Estimation in Tomato Leaves by VIS-NIR Non-Destructive Spectroscopy. Sensors, 2011; 6: 6411-6424.

[29] Retamales J B, Mena C, Lobos G. A regression analysis on factors affecting yield of highbush blueberries. Scientia Horticulturae, 2015; 186 7-14.

[30] Patel D M, Patel N M, Pandya N N. Gastroretentive drug delivery system of carbamazepine: Formulation optimization using simplex lattice design: A technical note. Aaps Pharmscitech, 2007; 1: 82-86.

[31] Karaman S, Yilmaz M T, Kayacier A. Simplex lattice mixture design approach on the rheological behavior of glucomannan based salep-honey drink mixtures: An optimization study based on the sensory properties. Food Hydrocolloids, 2011; 5: 1319-1326.

[32] Webber III C L, Whitworth J, Dole J. Kenaf (Hibiscus cannabinum L.) core as a containerized growth medium component. Industrial Crops and Products, 1999; 10: 97-105.

[33] Sparks D L, Page A L, Helmke P A, Leoppert R H, Soltanpour P N, Tabatabai M A, et al. Methods of Soil Analysis. Soil Sci. Soci. Am., Madison, Wisconsin.1996; pp.123-135.

[34] Coelho R F, Bouillard P. Multi-objective reliability-based optimization with stochastic metamodels. Evolutionary Computation, 2011; 4: 525-560.

[35] Deb K. Multi-objective genetic algorithms: problem difficulties and construction of test problems. Evolutionary Computation, 1999; 3: 205-230.

[36] Deb K. A fast and elitist multi-objective genetic algorithm: NSGA-II. IEEE Transactions on Evolutionary Computation, 2002; 6: 182-193.

[37] Ren Z Y, Liu Y L. Effects of different coir dust to perlite ratio substrate on growth and seedling raising effect of tomato seedling. Tianjin Agricultural Science, 2018; 5: 63-66. (in Chinese)

[38] Yang F, Tao G X, Sun Z H. Effects of different substrate composition on Capsicum frutescens L. var. shuanlaense seedlings. Anhui Agricultural Science, 2018; 28: 56-59. (in Chinese)

[39] Ozturk I, Karaman S, Baslar M, Cam M, Caliskan O, Sagdic O, et al. Aroma: sugar and anthocyanin profile of fruit and seed of mahlab (Prunus mahaleb L.): optimization of bioactive compounds extraction by simplex lattice mixture design. Food Anal. Methods, 2014; 7 : 761-773.

[40] Meinhart A D, da Silveira T F F, de Moraes M R, Petrarca M H, Silva L H, Oliveira $\mathrm{W} \mathrm{S}$, et al. Optimization of frying oil composition rich in essential fatty acids by mixture design. LWT-Food Sci. Technol., 2017; 84: 795-803.

[41] Baj T, Baryluk A, Sieniawska E. Application of mixture design for optimum antioxidant activity of mixtures of essential oils from, Ocimum basilicum, L., Origanum majorana L. and Rosmarinus officinalis, L. Industrial Crops and Products, 2018; 115: 52-61.

[42] Thangadurai K, Padmavathi K. Citrus canker disease detection using genetic algorithm in citrus plants. International Journal of Trend in Research and Development, 2015; 2(5): 434-437.

[43] Mansini R, Ogryczak W, Speranza M G. Twenty years of linear programming based portfolio optimization. European Journal of Operational Research, 2014; 234(2): 518-535.

[44] Kanagaraj G, Ponnambalam S G, Jawahar N. A hybrid cuckoo search and genetic algorithm for reliability-redundancy allocation problems. Computers\& Industrial Engineering, 2013; 66: 1115-1124. 